\title{
OMERACT-based fibromyalgia symptom subgroups: an exploratory cluster analysis
}

\author{
Ann Vincent ${ }^{*}$, Tanya L Hoskin², Mary O Whipple ${ }^{1}$, Daniel J Clauw ${ }^{3}$, Debra L Barton ${ }^{4}$, Roberto P Benzo ${ }^{5}$ \\ and David A Williams ${ }^{3}$
}

\begin{abstract}
Introduction: The aim of this study was to identify subsets of patients with fibromyalgia with similar symptom profiles using the Outcome Measures in Rheumatology (OMERACT) core symptom domains.

Methods: Female patients with a diagnosis of fibromyalgia and currently meeting fibromyalgia research survey criteria completed the Brief Pain Inventory, the 30-item Profile of Mood States, the Medical Outcomes Sleep Scale, the Multidimensional Fatigue Inventory, the Multiple Ability Self-Report Questionnaire, the Fibromyalgia Impact Questionnaire-Revised (FIQ-R) and the Short Form-36 between 1 June 2011 and 31 October 2011. Hierarchical agglomerative clustering was used to identify subgroups of patients with similar symptom profiles. To validate the results from this sample, hierarchical agglomerative clustering was repeated in an external sample of female patients with fibromyalgia with similar inclusion criteria.

Results: A total of 581 females with a mean age of 55.1 (range, 20.1 to 90.2) years were included. A four-cluster solution best fit the data, and each clustering variable differed significantly $(P<0.0001)$ among the four clusters. The four clusters divided the sample into severity levels: Cluster 1 reflects the lowest average levels across all symptoms, and cluster 4 reflects the highest average levels. Clusters 2 and 3 capture moderate symptoms levels. Clusters 2 and 3 differed mainly in profiles of anxiety and depression, with Cluster 2 having lower levels of depression and anxiety than Cluster 3, despite higher levels of pain. The results of the cluster analysis of the external sample $(n=478)$ looked very similar to those found in the original cluster analysis, except for a slight difference in sleep problems. This was despite having patients in the validation sample who were significantly younger $(P<0.0001)$ and had more severe symptoms (higher FIQ-R total scores $(P=0.0004)$ ).

Conclusions: In our study, we incorporated core OMERACT symptom domains, which allowed for clustering based on a comprehensive symptom profile. Although our exploratory cluster solution needs confirmation in a longitudinal study, this approach could provide a rationale to support the study of individualized clinical evaluation and intervention.
\end{abstract}

\section{Introduction}

Despite chronic, widespread pain being its cardinal symptom, fibromyalgia (FM) actually presents as a heterogeneous symptom complex that remains poorly understood $[1,2]$. In addition to pain, frequently occurring symptoms include fatigue, unrefreshing sleep, cognitive difficulties, headache, anxiety and depression [3]. Patients with FM often present with varying combinations and degrees of severity of these symptoms, which further complicates the

\footnotetext{
* Correspondence: vincent.ann@mayo.edu

'Division of General Internal Medicine, Mayo Clinic, 200 First Street SW, Rochester, MN 55902, USA

Full list of author information is available at the end of the article
}

ability to understand FM and makes its study challenging [4]. Stratifying patients into more homogeneous subgroups may facilitate better understanding of FM.

Several studies have reported homogeneous subgroups within samples of patients with FM discovered using the exploratory data-mining technique of cluster analysis [5-11]. Variables used to identify clusters in these studies have included sociodemographic variables, patient responses to self-report questionnaires, physiological and psychophysiological parameters, biomarkers, comorbidities and psychosocial functioning. For example, Giesecke et al. categorized patients with FM based on self-report measures and evoked pain testing [6]. In their study, 97 
patients were clustered into three subgroups: (1) extreme tenderness with normal mood, (2) low tenderness with moderate mood and (3) extreme tenderness with low mood. In another study, by de Souza et al., patients with FM were clustered into two groups based on their responses to the Fibromyalgia Impact Questionnaire (FIQ) [5]. In that study, both clusters reported similar levels of pain, fatigue and stiffness, but differed on severity of morning tiredness, anxiety and depression. Similarly, Docampo et al. clustered a large sample of Spanish patients with FM into three groups using dimensions of symptomatology, comorbidities and clinical scales. These subgroups included (1) low symptomatology and comorbidities, (2) high symptomatology and comorbidities and (3) high symptomatology and low comorbidities. These studies illustrate the symptom spectrum and symptom combinations that are possible in a heterogeneous sample of patients carrying the same diagnosis, and the results are consistent with those of Loevinger et al. and Wilson et al. $[7,10]$. It has also been reported that patients with FM belonging to different clusters may respond differently to pain rehabilitation. In separate studies, Verra et al. and Turk et al. demonstrated that (1) the same treatment can yield different responses, depending upon symptom constitution of a subgroup; and (2) tailoring treatment based on symptoms could improve treatment efficacy $[9,12]$.

Although a wide range of variables have been used to identify clusters of patients with FM, few studies have included a comprehensive symptom profile. The Outcome Measures in Rheumatology (OMERACT) FM working group recommended 12 domains (including both symptoms and biomarkers) to be used in FM studies: pain, fatigue, sleep disturbance, depression, anxiety, stiffness, dyscognition, patient global impression of health, multidimensional functioning, tenderness, cerebrospinal fluid (CSF) biomarkers and pain-related neuroimaging markers (if available) [13]. The OMERACT domains provide an all-inclusive list of symptoms that may be used to better understand FM. Our objective in the present study was to identify clusters within a heterogeneous sample of patients with FM using OMERACT symptom domains assessed with validated, self-report questionnaires. We then sought to validate our cluster results in an independent sample of patients with FM.

\section{Methods}

\section{Ethics statement}

The study was approved by the Mayo Clinic Institutional Review Board, and all participants provided written informed consent.

Data for the exploratory cluster analysis were derived from survey responses of a sample of patients randomly selected from an existing FM registry that has been described previously [14]. Briefly, the FM registry is composed of patients who have been seen at the Mayo Clinic with a diagnosis of FM since 1 January 2000 and have consented to enrollment in the registry. Participants were invited to participate by postal survey and completed a questionnaire package that included the Brief Pain Inventory (BPI) [15], the 30-item Profile of Mood States (30-item POMS) [16], the Medical Outcomes Study Sleep Scale (MOS-Sleep) [17], the Multidimensional Fatigue Inventory (MFI-20) [18], the Multiple Ability Self-report Questionnaire (MASQ) [19], the Revised Fibromyalgia Impact Questionnaire (FIQ-R) [20] and the 36-item Short Form survey (SF-36) [21]. All instruments included are considered appropriate in meaningfully characterizing the multiple facets of FM as described by Williams and Arnold [22].

\section{Participants}

Only female respondents who met FM research survey criteria were included in the exploratory cluster analysis [23]. Male respondents were excluded $(n=50)$, because the number of males in our sample was too small to analyze meaningfully, given evidence which suggests that the symptom experience for males with FM may differ from that of females [24-27].

\section{Measures}

OMERACT-recommended symptom domains were assessed using either total scores or subscale scores of the questionnaires described below [28]. Our goal was to select the scale or subscale that best represented the OMERACT symptom domain while eliminating the possibility of item overlap between symptom domains and clustering variables. For example, in the case of MFI-20, which includes physical fatigue, mental fatigue (related to cognition) and reduced motivation (related to mood), we purposefully selected physical fatigue to keep this symptom construct distinct. The subscales selected for each domain are shown in Table 1. Of the core OMERACT symptom domains, all except objective tenderness and patient global impression of change to treatment (which was not applicable, given that there was no treatment comparison) were available. Table 1 lists the measures used to operationalize OMERACT domains (that is, used for clustering) and those measures used as descriptive clinical outcomes against which the clusters were evaluated.

\section{Pain (Brief Pain Inventory)}

The BPI is a 15 -item, validated self-report measure of chronic, non-cancer-related pain and assesses presence of pain, pain severity and pain interference. It yields two subscales: pain severity and pain interference. Scores on pain severity and pain interference range from 0 to 10 , with higher scores indicating greater pain. Internal consistency for the Pain Severity score is 0.85 and that 


\begin{tabular}{ll}
$\begin{array}{l}\text { Table } \mathbf{1} \text { Scales and subscales representing OMERACT } \\
\text { domains }\end{array}$ \\
\hline OMERACT domain & Scale/Subscale selected \\
\hline Pain & BPI Pain Severity \\
Fatigue & MFI Physical Fatigue \\
Function & FIQ Function \\
Sleep disturbance & MOS Sleep Problems Index II \\
Depression & POMS Depression Dejection \\
Dyscognition & MASQ Total \\
Anxiety & POMS Tension Anxiety \\
Stiffness & FIQ Stiffness \\
Tenderness & NA \\
Patient global assessment & NA \\
CSF biomarkers & NA \\
Functional Imaging & NA \\
\hline
\end{tabular}

a BPI, Brief Pain Inventory; CSF, Cerebrospinal fluid; FIQ, Fibromyalgia Impact Questionnaire; MASQ, Multiple Ability Self-report Questionnaire; MFI,

Multidimensional Fatigue Inventory; MOS-Sleep, Medical Outcomes Study Sleep Scale; NA, Not applicable; OMERACT, Outcome Measures in Rheumatology; POMS, Profile of Mood States.

for the Interference scale is 0.88 [29]. The BPI has been used in FM clinical trials and is considered an appropriate measure of pain in FM [22,30-33]. For this analysis, we selected the Pain Severity subscale to represent the OMERACT symptom domain of pain.

\section{Depression and anxiety (30-item Profile of Mood States)}

The 30-item POMS is a validated self-report measure of mood. It yields six subscales: (1) depression-dejection, (2) tension-anxiety, (3) fatigue-inertia, (4) vigor-activity, (5) anger-hostility and (6) confusion-bewilderment. Scores on each subscale range from 0 to 20 , with higher scores indicating worse symptoms on all scales, except for the vigor-activity scale, on which lower scores indicate worse symptoms. Although the majority of publications describing POMS in FM have been based on the 65-item instrument, the 30-item POMS has an internal consistency of 0.69 to 0.88 and is considered a superior measure in general [34-36]. For this analysis, we selected the DepressionDejection and Tension-Anxiety subscales to represent the OMERACT symptom domains of depression and anxiety.

\section{Sleep disturbance (Medical Outcomes Study-Sleep scale)}

The MOS-Sleep scale is a 12-item, validated, self-report measure that assesses six dimensions of sleep: (1) sleep disturbance, (2) sleep adequacy, (3) sleep quantity, (4) somnolence, (5) snoring and (6) awakening with shortness of breath or headache. It yields two summary indices: the Sleep Problems Index I (six items) and the Sleep Problems Index II (nine items). Scores on dimensions and summary indices range from 0 to 100 , with higher scores indicating poorer sleep. The MOS-Sleep scale has an internal consistency of 0.7 in patients with FM [17]. The MOS-Sleep scale has been used in many FM clinical trials and is considered an appropriate measure of sleep in FM [37-39]. For this analysis, we selected the Sleep Problems Index II to represent the OMERACT symptom domain of sleep disturbance.

\section{Fatigue (Multidimensional Fatigue Inventory)}

The MFI-20 is a 20 -item validated self-report measure of fatigue and assesses general fatigue, physical fatigue, reduced activity, reduced motivation and mental fatigue [18]. Subscale scores range from 4 to 20, with higher scores indicating greater fatigue. It has an internal consistency of 0.93 [40]. The MFI-20 has been used in clinical trials of FM and chronic pain and is an appropriate measure of fatigue in FM $[31,41,42]$. For this analysis, we selected the MFI Physical Fatigue subscale to represent the OMERACT symptom domain of fatigue.

\section{Dyscognition (Multiple Ability Self-report Questionnaire)}

The MASQ is a 38-item self-report measure and assesses five cognitive domains: language, visuoperceptual, verbal memory, visual memory and attention [19]. Scores on the cognitive domains range from 0 to 30 or 0 to 40 , and the maximum total score is 190. Higher scores indicate greater perceived difficulties with cognition. The MASQ has an internal consistency of 0.92 . It has been used in several FM clinical trials to measure change in perceived cognition [43-45]. For this analysis, we selected the MASQ total to represent the OMERACT symptom domain of dyscognition.

\section{Stiffness (Revised Fibromyalgia Impact Questionnaire)}

The FIQ-R is a 21-item, validated self-report measure that assesses the symptoms, physical functioning and overall impact of FM [20]. Scores range from 0 to 100, with higher scores indicating greater symptom burden. It has an internal consistency of 0.95 and is the most commonly used outcome measure in FM clinical trials $[22,37,46,47]$. For this analysis, we selected the FIQ-R Stiffness question to represent the OMERACT symptom domain of stiffness. Additionally, FIQ-R total scores (not used in the clustering) were compared across the subgroups identified by cluster analysis.

\section{SF-36}

The SF-36 version 2 is a 36 -item, validated self-report measure that assesses disease burden [21]. It consists of eight subscales and two summary scores (physical and mental components). Component scores range from 0 to 100 , with higher scores indicating better health. The SF-36 has an internal consistency of 0.9 and has been used in FM clinical trials [48-50]. Similarly to the FIQ-R total score, the SF-36 component scores were not 
included in the clustering, but were used for comparison of symptom levels across clusters.

\section{Fibromyalgia research survey criteria}

The FM research survey criteria have been validated for use in epidemiologic and survey studies [23]. It yields a widespread pain index (WPI) score (range, 0 to 19) and a symptom severity (SS) score of (0 to 12 ). Patients are classified as meeting FM research survey criteria if their WPI was $\geq 7$ and SS was $\geq 5$ or if their WPI was between 3 and $6 \mathrm{SS} \geq 9$. Higher scores indicate more severe symptoms. WPI and SS scores were also compared across clusters.

\section{Participants and measures in the external validation sample}

To provide an external validation measure, we chose a separate sample of patients with FM $(n=478)$ who participated in the FM Treatment Program at Mayo Clinic but were not yet enrolled in the FM registry. Patients who participate in this program complete a comprehensive package of questionnaires. This comprehensive package includes the same measures used in the exploratory cluster analysis, with the exception of POMS and BPI. In this sample, in place of POMS, the severity of depression and anxiety were assessed with Patient Health Questionnaire (PHQ-9) and Generalized Anxiety Disorder 7-item scale (GAD-7), both of which are clinically validated measures [51-53]. Although BPI was not part this package, the Pain subscale of the FIQ provides a similar measure of pain severity and is considered appropriate to meaningfully characterize pain in FM, as described by Williams and Arnold [22]. Similarly to the exploratory cluster sample, only female participants who met FM Research Survey Criteria were included. Importantly, medication use at the time of the survey (that is, the clinic visit) was available for participants included in the validation sample, whereas such data were not available in the original registry survey-based cluster sample.

\section{Statistical methods}

Cluster analysis variables were standardized by subtracting the variable mean from each individual observation and dividing by the standard deviation. Hierarchical agglomerative clustering with Ward's method and squared Euclidean distances was used on the standardized data. The number of clusters was chosen by examining the dendrogram and based on clinical interpretability and usefulness. Clusters were subsequently compared on variables of interest using analysis of variance, followed by pairwise comparisons between clusters when the omnibus test was significant (that is, $P<0.05$ ). For pairwise comparisons, a Bonferroni adjustment to the 0.05 significance level was applied when interpreting $P$-values; because our analysis resulted in four clusters, and thus six pairwise comparisons, the Bonferroni $P$-value for interpreting pairwise significance tests was $0.05 / 6=$ 0.0083 . Analysis was performed using $\mathrm{R}$ [54] statistical software (version 2.15.0; $\mathrm{R}$ Foundation for Statistical Computing, Vienna, Austria) and JMP (version 10.0.0; SAS Institute, Cary, NC, USA). The same methods used for cluster analysis and comparison across clusters were applied to the validation sample. Because standardized variables (that is, $\mathrm{z}$-scores) rather than raw scales were used for the cluster analysis, we felt it was reasonable to interpret and compare results from the validation and original samples, although a few of the specific instruments to measure individual symptoms differed between the two samples, as described above.

\section{Results}

\section{Exploratory cluster analysis}

Of the 655 patients who met the inclusion criteria for the exploratory cluster analysis, 74 patients had missing data for one or more of the cluster analysis variables, yielding a final sample size of 581 patients for analysis. Demographic and symptom scales for the sample are summarized in Table 2.

Hierarchical agglomerative clustering on the standardized variables corresponding to fatigue, sleep, pain, function, stiffness, dyscognition, depression and anxiety resulted in a dendrogram that suggested meaningful information when the data were examined with between three and four clusters. The four-cluster solution largely divided the sample into severity levels, with cluster 1 reflecting the lowest average levels across all symptoms, cluster 4 reflecting the highest average levels across all symptoms and clusters 2 and 3 capturing generally moderate symptom levels. An important distinction between clusters 2 and 3 was their different profiles on the mental aspects of the disease, as cluster 2 clearly had lower levels of depression and anxiety than did cluster 3, despite cluster 2's having somewhat higher levels of pain, stiffness, dysfunction, sleep disturbance and fatigue (Figure 1A). Considering a three-cluster solution, clusters 1 and 2 would have remained together, which we felt would miss a clinically important difference revealed by the fourcluster solution because these two subgroups have significantly different levels of fatigue, sleep, pain, stiffness, function and dyscognition despite their similar levels of negative mood. Thus, the four-cluster solution was used for the subsequent analyses described below.

\section{Characterizing clusters}

The four clusters were similar in size, with clusters 1 to 4 comprising $26 \%, 29 \%, 23 \%$ and $21 \%$ of the samples, respectively. Each of the clustering variables differed significantly between the four clusters (Table 3 ). The pairwise comparisons also showed significant differences 
Table 2 Patient characteristics of 581 patients included in cluster analysis $^{a}$

\begin{tabular}{|c|c|c|}
\hline Characteristics & $\begin{array}{l}\text { Mean (SD) or } \\
n(\%)\end{array}$ & Median (range) \\
\hline Age, yr & $55.1(12.4)$ & 55.6 (20.1 to 90.2$)$ \\
\hline \multicolumn{3}{|l|}{ Race } \\
\hline White & $531(91.4)$ & \\
\hline Other & $10(1.7)$ & \\
\hline Missing data & $40(6.9)$ & \\
\hline
\end{tabular}

Education

Less than high school
High school graduate or GED
Some college or 2-year degree
4-year college graduate
Postgraduate studies
Missing data
Employment status
Employed
Retired
Work-disabled

$13(2.2)$

$122(21.0)$

$225(38.7)$

$92(15.8)$

86 (14.8)

$43(7.4)$

$206(35.4)$

$124(21.3)$

$109(18.8)$

Other (student, full-time homemaker, 69 (11.9) other)

$\begin{array}{ll}\text { Unemployed } & 51(8.8) \\ \text { Missing data } & 22(3.8) \\ \text { Marital status } & \end{array}$

$\begin{array}{ll}\text { Married/committed relationship } & 435(74.9) \\ \text { Divorced/separated } & 55(9.4) \\ \text { Single } & 44(7.6) \\ \text { Widowed } & 25(4.3) \\ \text { Missing data } & 22(3.8) \\ & 30.1(7.6)\end{array}$

$\mathrm{BMI}, \mathrm{kg} / \mathrm{m}^{2}$

FM research survey

$\begin{array}{lll}\text { WPI } & 12.6(3.8) & 13 \text { (3 to } 19) \\ \text { SS } & 8.9(2.0) & 9(5 \text { to } 12) \\ \text { BPI } & 5.1(1.8) & 5.3(0.3 \text { to } 10) \\ \text { Pain severity } & 5.7(2.3) & 5.9(0 \text { to } 16.3) \\ \text { Pain interference } & 55.1(18.9) & 56.7(8.9 \text { to } 97.8) \\ \text { MOS-Sleep Problems Index II } & & \\ \text { POMS } & 6.8(5.1) & 6(0 \text { to } 20) \\ \text { Depression-Dejection } & 7.2(4.8) & 7(0 \text { to } 20) \\ \text { Tension-Anxiety } & & \\ \text { MFI-20 } & 17.3(2.6) & 18(7 \text { to } 20) \\ \text { General } & 15.8(3.6) & 17(4 \text { to } 20) \\ \text { Physical } & 14.6(3.9) & 15(4 \text { to } 20) \\ \text { Reduced activity } & 12.4(3.8) & 12(4 \text { to } 20) \\ \text { Reduced motivation } & \end{array}$

Table 2 Patient characteristics of 581 patients included in cluster analysis ${ }^{\mathrm{a}}$ (Continued)

\begin{tabular}{lll}
\hline Mental & $13.2(4.2)$ & $13(4$ to 20$)$ \\
Total & $73.3(14.0)$ & $74(29$ to 100$)$ \\
MASQ & $95.4(22.0)$ & $94(49$ to 162$)$ \\
FIQ-R total & $56.0(18.7)$ & $57.5(5$ to 96.2$)$ \\
$\quad$ Stiffness & $7.2(2.2)$ & $8(0$ to 10$)$ \\
SF-36 & & \\
$\quad$ Physical & $30.3(8.6)$ & $29.8(8.3$ to 52.9$)$ \\
Mental & $40.2(12.6)$ & $40.5(7.5$ to 66.8$)$ \\
\hline
\end{tabular}

${ }^{\mathrm{a} B M I}$, Body mass index; BPI, Brief Pain Inventory; FIQ-R, Revised Fibromyalgia Impact Questionnaire; FM, Fibromyalgia; GED, General educational development; MASQ, Multiple Ability Self-Report Questionnaire ; MFI, Multidimensional Fatigue Inventory; MOS-Sleep, Medical Outcomes Study Sleep Scale; POMS, Profile of Mood States; SF-36, Medical Outcomes Study 36-item Short Form survey; SS, Symptom severity; WPI, Widespread pain index.

of each cluster from every other cluster on all symptoms, with a few exceptions. Clusters 2 and 3 did not differ significantly on sleep problems $(P=0.16)$, and clusters 1 and 2 did not differ significantly on either depression $(P=0.23)$ or anxiety $(P=0.26)$.

Clinically relevant variables external to the cluster creation were compared across clusters, and the results are given in Table 4. FIQ Impact was significantly different for each cluster (all $P$-values $<0.0001$ ), except for cluster 2 versus cluster 3 , which had very similar levels (mean, 11.5 vs 11.7; $P=0.76$ ). Similarly, clusters 2 and 3 had similar FIQ Total scores (mean, 58.1 vs 57.7). This underscores the point that both clusters have a globally "moderate" level of symptoms, although the drivers appear to be more physical in cluster 2 and more mental in cluster 3. The SF-36 mental composite score differed significantly for each cluster, and the physical composite score differed for every cluster comparison except cluster 2 versus cluster 4 (mean, 26.2 vs $25.6 ; P=0.53$ ).

Interestingly, patients in clusters 1 and 2 , who had the lowest levels of depression and anxiety, were also significantly older than those in clusters 3 and 4. Body mass index (BMI) varied somewhat across clusters, but the only pairwise comparison that reached statistical significance was for cluster 1 versus 2 (mean, 28.6 vs 31.5; $P=0.0008)$.

\section{External sample validation}

A total of 478 female patients who met the FM research survey criteria were included in the validation sample. The mean age of the participants was $46.5( \pm 13.1)$ years, mean BMI was $30.2( \pm 7.8) \mathrm{kg} / \mathrm{m}^{2}$ and the mean FIQ Total score was $60.0( \pm 17.4)$. Compared to the original sample, the validation sample was significantly younger $(P<0.0001)$, had a similar BMI $(P=0.74)$ and had a higher FIQ Total score $(P=0.0004)$. 


\section{A Original Cluster Sample}
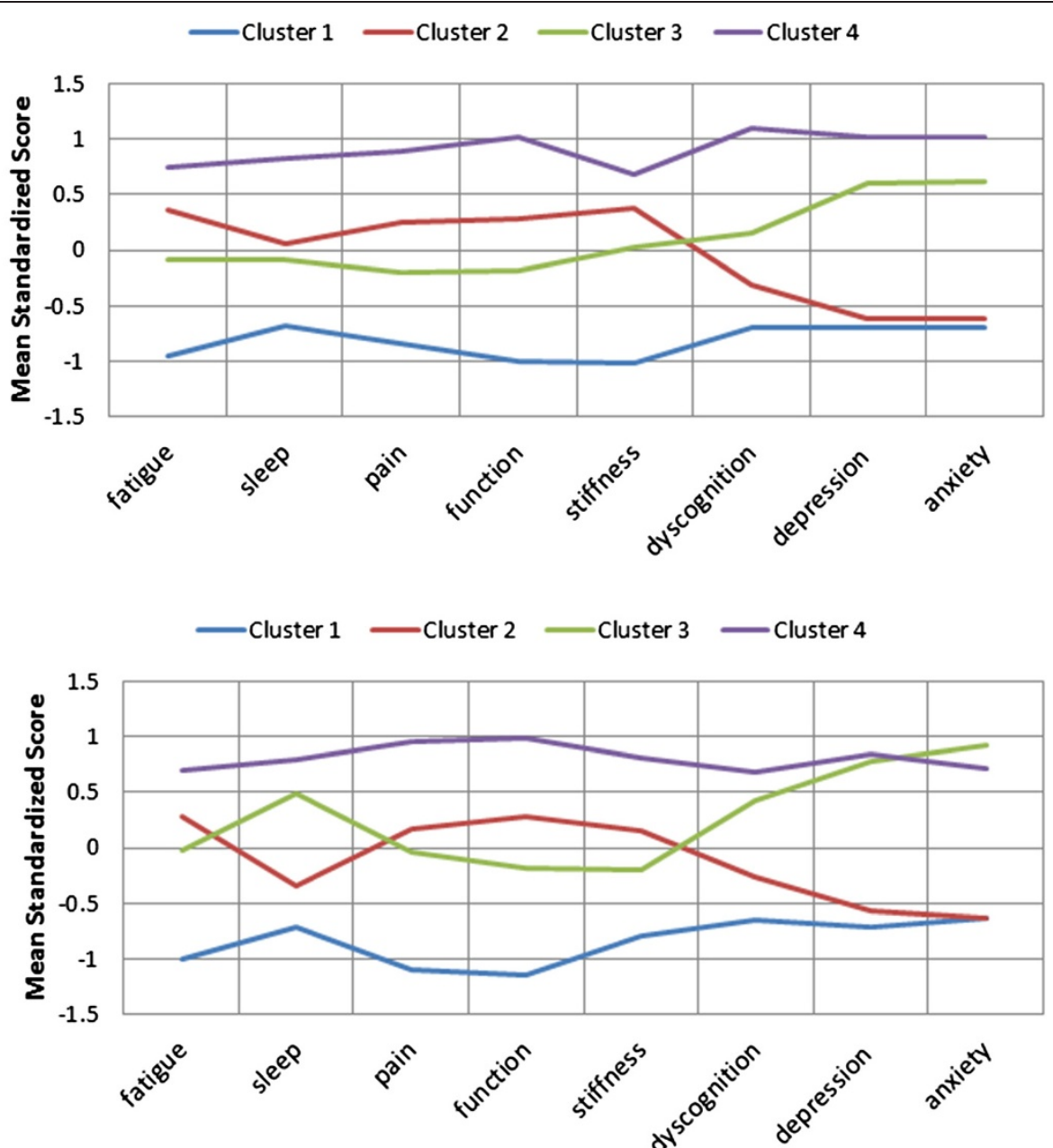

Figure 1 Cluster profiles plot for each of the two study samples. (A) Original sample. (B) External validation sample.

As with the exploratory cluster analysis, the dendrogram of the hierarchical clustering solution for the validation sample suggested four clusters. The four clusters comprised $24 \%, 32 \%, 21 \%$ and $23 \%$ of the sample, respectively. The four-cluster solution from the validation sample similarly divided the sample into severity levels, with cluster 1 demonstrating the lowest symptom severity and cluster 4 having the highest symptom severity (Figure 1B). Similarly to the exploratory cluster analysis, each of the clustering variables differed significantly among the four clusters (Table 5). The pairwise comparisons also showed significant differences of each cluster from every other cluster on all symptoms, with a few exceptions. Clusters 1 and 2 did not differ significantly on anxiety $(P=0.96)$ or depression $(P=0.07)$. Clusters 3 and 4 did not differ significantly on depression $(P=0.53)$. The symptom profiles looked very similar to those found in the original cluster analysis sample, with a slight difference for sleep problems, which did not differ significantly between clusters 2 and 3 in the original sample but were significantly worse for cluster 3 vs cluster 2 in the validation sample.

The FIQ-Total and SF-36 scores serve as external benchmarks for the cluster differentiation. As with the original cluster sample, these scores are consistent with our interpretation of the clusters, specifically that cluster 1 shows a globally low symptom level, cluster 4 shows a globally high symptom level and clusters 2 and 3 are both generally moderate at the global level based on FIQ-Total scores (Table 6), whereas the SF-36 Mental component score differentiated clusters 2 and 3.

The percentage of patients on work disability also differed significantly between clusters $(P<0.0001)$. Cluster 1 had the lowest percentage of patients on work disability (9.6\%), clusters 2 and 3 had a greater number of patients on work disability (18.6\% and $19.1 \%$ of patients, respectively) and cluster 4 had the greatest percentage of patients on work disability (33.1\%). 
Table 3 Cluster analysis variables compared across clusters ${ }^{\mathrm{a}}$

\begin{tabular}{|c|c|c|c|c|c|}
\hline Measure & Cluster $1^{b}(n=150)$ & Cluster $2^{c}(n=171)$ & Cluster $3^{d}(n=136)$ & Cluster $4^{e}(n=124)$ & $P$-value ${ }^{f}$ \\
\hline MFI physical fatigue & $12.5(3.7)$ & $17.1(2.4)$ & $15.5(2.8)$ & $18.5(1.7)$ & $<0.0001$ \\
\hline MOS-Sleep Problems Index II & $42.4(16.5)$ & $56.2(17.2)$ & $53.5(15.4)$ & $70.8(15.5)$ & $<0.0001$ \\
\hline BPI pain severity & $3.6(1.4)$ & $5.5(1.4)$ & $4.7(1.5)$ & $6.6(1.2)$ & $<0.0001$ \\
\hline MASQ & $80.1(15.7)$ & $88.5(16.9)$ & $98.8(17.8)$ & $119.5(17.4)$ & $<0.0001$ \\
\hline FIQ function & $7.1(4.8)$ & $16.2(5.7)$ & $12.9(5.2)$ & $21.5(4.0)$ & $<0.0001$ \\
\hline FIQ stiffness & $5.0(2.2)$ & $8.0(1.4)$ & $7.2(1.8)$ & $8.7(1.1)$ & $<0.0001$ \\
\hline POMS tension-anxiety & $3.9(3.1)$ & $4.3(2.6)$ & $10.2(3.6)$ & $12.1(3.8)$ & $<0.0001$ \\
\hline POMS Depression-Dejection & $3.2(3.2)$ & $3.7(2.5)$ & $10.0(3.7)$ & $12.1(4.5)$ & $<0.0001$ \\
\hline
\end{tabular}

${ }^{\mathrm{a}}$ Data are mean \pm SD. BPI, Brief Pain Inventory; FIQ, Fibromyalgia Impact Questionnaire; MASQ, Multiple Ability Self-report Questionnaire; MFI, Multidimensional Fatigue Inventory; MOS-Sleep Problems Index II, Medical Outcomes Study Sleep scale; POMS, Profile of Mood States; SF-36, Medical Outcomes Study 36-item Short Form survey. ${ }^{b}$ Low symptom intensity. ${ }^{c}$ Moderate symptoms, low negative mood. ${ }^{d}$ Moderate symptoms, higher negative mood and dyscognition. ${ }^{e}$ High symptom intensity. ${ }^{f} P$-values reported are from the omnibus analysis of variance test across the four clusters. Pairwise comparisons between clusters also showed significant differences in all cases, except for the following: cluster 2 versus cluster 3 MOS-Sleep Problems Index II $(P=0.16)$ and cluster 1 versus cluster 2 with respect to both POMS Tension-Anxiety $(P=0.26)$ and Depression-Dejection $(P=0.23)$.

Table 4 Nonclustering variables compared across clusters ${ }^{a}$

\begin{tabular}{|c|c|c|c|c|c|}
\hline Variables & Cluster $1^{b}(n=150)$ & Cluster $2^{c}(n=171)$ & Cluster $3^{d}(n=136)$ & Cluster $4^{\mathrm{e}}(n=124)$ & $P$-value ${ }^{f}$ \\
\hline Age, yr & $57.0(14.5)$ & $57.6(11.3)$ & $52.5(12.4)$ & $52.2(9.7)$ & $<0.0001$ \\
\hline $\mathrm{BMl}, \mathrm{kg} / \mathrm{m}^{2}$ & $28.6(7.6)$ & $31.5(7.8)$ & $29.2(6.9)$ & $30.8(7.8)$ & 0.0030 \\
\hline \multicolumn{6}{|l|}{ FM research survey } \\
\hline WPI & $11.1(3.5)$ & $12.8(3.7)$ & $12.5(3.6)$ & $14.3(3.5)$ & $<0.0001$ \\
\hline SS & $7.6(1.8)$ & $8.7(1.8)$ & $9.1(1.8)$ & $10.5(1.4)$ & $<0.0001$ \\
\hline BPI Pain interference & $3.4(1.9)$ & $5.9(1.8)$ & $5.9(1.6)$ & $7.9(1.3)$ & $<0.0001$ \\
\hline \multicolumn{6}{|l|}{$\mathrm{MFI}-20$} \\
\hline General & $15.0(2.8)$ & $17.8(2.1)$ & $17.5(2.2)$ & $19.0(1.6)$ & $<0.0001$ \\
\hline Reduced activity & $11.7(3.9)$ & $15.0(3.4)$ & $14.6(3.4)$ & $17.6(2.3)$ & $<0.0001$ \\
\hline Reduced motivation & $9.8(3.1)$ & $12.1(3.5)$ & $12.9(3.3)$ & $15.5(2.9)$ & $<0.0001$ \\
\hline Mental & $10.5(3.8)$ & $12.4(3.8)$ & $13.9(3.9)$ & $16.6(2.9)$ & $<0.0001$ \\
\hline Total & $59.3(11.8)$ & $74.5(10.5)$ & $74.4(10.4)$ & $87.1(7.1)$ & $<0.0001$ \\
\hline \multicolumn{6}{|l|}{ SF-36 } \\
\hline Physical $^{9}$ & $36.5(8.3)$ & $26.2(7.3)$ & $33.1(6.8)$ & $25.6(6.4)$ & $<0.0001$ \\
\hline Mental $\left.\right|^{9}$ & $48.9(9.3)$ & $45.4(10.1)$ & $34.5(9.3)$ & $28.6(10.3)$ & $<0.0001$ \\
\hline \multicolumn{6}{|l|}{$\mathrm{FIQ}$} \\
\hline Tenderness & $5.8(2.8)$ & $7.8(2.3)$ & $7.7(2.3)$ & $8.8(1.6)$ & $<0.0001$ \\
\hline Impact & $5.7(4.5)$ & $11.5(5.1)$ & $11.7(4.7)$ & $16.3(3.7)$ & $<0.0001$ \\
\hline Total & $34.3(11.7)$ & $58.1(13.0)$ & $57.7(10.8)$ & $77.7(8.6)$ & $<0.0001$ \\
\hline \multicolumn{6}{|l|}{ POMS } \\
\hline Anger-Hostility & $3.0(3.3)$ & $3.2(2.7)$ & $6.8(4.2)$ & $8.6(4.4)$ & $<0.0001$ \\
\hline Vigor-Activity $^{9}$ & $6.5(3.6)$ & $3.9(3.1)$ & $3.8(2.8)$ & $2.4(2.6)$ & $<0.0001$ \\
\hline Fatigue-Inertia & $10.2(3.7)$ & $13.6(3.6)$ & $14.7(3.1)$ & $16.9(2.6)$ & $<0.0001$ \\
\hline Confusion-Bewilderment & $4.7(2.9)$ & $6.1(2.7)$ & $9.1(2.8)$ & $12.7(3.2)$ & $<0.0001$ \\
\hline
\end{tabular}

${ }^{\mathrm{a}}$ Data are mean \pm SD. BMI, Body mass index; BPI, Brief Pain Inventory; FIQ, Fibromyalgia Impact Questionnaire; FM, Fibromyalgia; MFI, Multidimensional Fatigue Inventory; POMS, Profile of Mood States; SF-36, 36-item Medical Outcomes Study Short Form health survey; SS, Symptom severity; WPI, Widespread pain index. ${ }^{b}$ Low symptom intensity. ${ }^{\mathrm{C}}$ Moderate symptoms, low negative mood. ${ }^{\mathrm{d}}$ Moderate symptoms, higher negative mood and dyscognition. ${ }^{\mathrm{e}}$ High symptom severity. ${ }^{f} P$-values are from the omnibus analysis of variance test across the four clusters. Pairwise comparisons between clusters also showed significant differences in all cases, except for the following: cluster 1 versus cluster 2 with respect to age $(P=0.62)$, cluster 1 versus cluster 3 with respect to BMI ( $P=0.51)$, cluster 2 versus cluster 4 with respect to both BMI $(P=0.49)$ and SF-36 Physical $(P=0.53)$ and cluster 3 versus cluster 4 with respect to both age $(P=0.86)$ and BMI $(P=0.08)$. Differences between clusters 2 and 3 were not significant for all variables, except age, BMI, MFI Reduced Motivation, MFI Mental Fatigue, SF-36 Physical, SF-36 Mental, POMS Fatigue-Inertia and POMS Confusion-Bewilderment. 'ower scores are worse for these scales. 
Table 5 Cluster analysis variables compared across clusters in the validation sample ${ }^{a}$

\begin{tabular}{|c|c|c|c|c|c|}
\hline Measure & Cluster $1^{b}(n=116)$ & Cluster $2^{c}(n=154)$ & Cluster $3^{d}(n=100)$ & Cluster $4^{e}(n=108)$ & $P$-value ${ }^{f}$ \\
\hline MFI physical fatigue & $13.0(3.9)$ & $17.5(2.3)$ & $16.4(2.7)$ & $18.9(1.8)$ & $<0.0001$ \\
\hline MOS-Sleep problems Index II & $46.1(15.9)$ & $52.9(16.3)$ & $68.5(14.8)$ & $74.1(12.3)$ & $<0.0001$ \\
\hline FIQ pain & $4.8(1.5)$ & $7.1(1.3)$ & $6.7(1.2)$ & $8.5(0.9)$ & $<0.0001$ \\
\hline MASQ & $82.7(18.1)$ & $91.3(21.3)$ & $107.0(17.1)$ & $112.8(19.5)$ & $<0.0001$ \\
\hline FIQ function & $7.0(4.8)$ & $17.4(5.2)$ & $14.0(5.2)$ & $22.6(3.5)$ & $<0.0001$ \\
\hline FIQ stiffness & $5.4(2.2)$ & $7.5(1.8)$ & $6.7(2.2)$ & $8.9(1.0)$ & $<0.0001$ \\
\hline GAD-7 & $5.0(4.1)$ & $5.0(3.7)$ & $14.2(4.0)$ & $13.0(4.7)$ & $<0.0001$ \\
\hline PHQ-9 & $8.6(4.1)$ & $9.5(3.2)$ & $17.1(4.5)$ & $17.4(4.3)$ & $<0.0001$ \\
\hline
\end{tabular}

${ }^{a} N=478$ total validation sample. Data are $n(\%)$. FIQ, Revised Fibromyalgia Impact Questionnaire; GAD-7, Generalized Anxiety Disorder 7-item scale; MASQ, Multiple Ability Self-report Questionnaire; MFI, Multidimensional Fatigue Inventory; MOS-Sleep Problems Index II, Medical Outcomes Study Sleep Scale; PHQ-9, Patient Health Questionnaire. ${ }^{b}$ Low symptom intensity. ${ }^{C}$ Moderate symptoms, low negative mood. ${ }^{d}$ Moderate symptoms, higher negative mood and dyscognition. ${ }^{\mathrm{e}} \mathrm{High}$ symptom intensity. ${ }^{\mathrm{f}} \mathrm{P}$-values are from the omnibus analysis of variance test across the four clusters. Pairwise comparisons between clusters also showed significant differences in all cases, except for the following: cluster 1 versus cluster 2 in regard to GAD-7 $(P=0.96)$ and PHQ-9 $(P=0.07)$ and cluster 3 versus cluster 4 with respect to PHQ-9 $(P=0.53)$.

Table 6 Nonclustering variables, including medication use, compared across clusters in external sample ${ }^{a}$

\begin{tabular}{|c|c|c|c|c|c|}
\hline Variables & Cluster $1^{b}(n=116)$ & Cluster $2^{c}(n=154)$ & Cluster $3^{d}(n=100)$ & Cluster $4^{\mathrm{e}}(n=108)$ & $P$-value ${ }^{f}$ \\
\hline Age, yr & $49.3(15.2)$ & $47.4(13.2)$ & $45.1(12.1)$ & $43.7(10.9)$ & 0.0074 \\
\hline $\mathrm{BMI}, \mathrm{kg} / \mathrm{m}^{2}$ & $29.1(6.7)$ & $31.3(8.5)$ & $29.7(8.3)$ & $30.3(7.2)$ & 0.14 \\
\hline \multicolumn{6}{|l|}{ FM research survey } \\
\hline WPI & $12.1(3.6)$ & $13.2(3.7)$ & $12.9(3.6)$ & $14.3(3.3)$ & $<0.0001$ \\
\hline SS & $7.1(1.7)$ & $8.1(1.7)$ & $9.3(1.5)$ & $9.6(1.5)$ & $<0.0001$ \\
\hline \multicolumn{6}{|l|}{ SF-36 } \\
\hline Physical $^{9}$ & $36.3(7.4)$ & $26.2(7.0)$ & $31.7(6.8)$ & $24.6(5.7)$ & $<0.0001$ \\
\hline Mental $^{9}$ & $45.0(10.6)$ & $42.6(10.2)$ & $29.7(9.8)$ & $31.6(9.8)$ & $<0.0001$ \\
\hline FIQ total & $37.9(10.7)$ & $61.3(10.8)$ & $62.4(9.2)$ & $79.3(7.9)$ & $<0.0001$ \\
\hline \multicolumn{6}{|l|}{ Medications } \\
\hline SNRI & $26(22.4)$ & $37(24.0)$ & $34(34.0)$ & $48(44.4)$ & 0.0007 \\
\hline TCA & $12(10.3)$ & $20(13.0)$ & $14(14.0)$ & $10(9.3)$ & 0.66 \\
\hline$a-2-\delta$ ligand & $23(19.8)$ & $44(28.6)$ & $24(24.0)$ & $32(29.6)$ & 0.29 \\
\hline SSRI & $22(19.0)$ & $36(23.4)$ & $29(29.0)$ & $26(24.1)$ & 0.39 \\
\hline Others $^{\mathrm{h}}$ & $17(14.7)$ & $18(11.7)$ & $23(23.0)$ & $18(16.7)$ & 0.11 \\
\hline $\begin{array}{l}\text { Any medication that could be used to } \\
\text { treat depression (above) }\end{array}$ & $72(62.1)$ & $105(68.2)$ & $76(76.0)$ & $83(76.9)$ & 0.0488 \\
\hline Tramadol & $12(10.3)$ & $22(14.3)$ & $15(15.0)$ & $20(18.5)$ & 0.38 \\
\hline Skeletal muscle relaxants & $20(17.2)$ & $43(27.9)$ & $24(24.0)$ & $33(30.6)$ & 0.10 \\
\hline Opioids & $21(18.1)$ & $51(33.1)$ & $28(28.0)$ & $57(52.8)$ & $<0.0001$ \\
\hline Benzodiazepines & $22(19.0)$ & $32(20.8)$ & $49(49.0)$ & $48(44.4)$ & $<0.0001$ \\
\hline Sleep aids & $26(22.4)$ & $48(31.2)$ & $30(30.0)$ & $39(36.1)$ & 0.16 \\
\hline Total number of medications, mean $( \pm \mathrm{SD})$ & $1.9(1.6)$ & $2.5(1.9)$ & $3.1(2.1)$ & $3.4(2.0)$ & $<0.0001$ \\
\hline $\begin{array}{l}\text { Use of one or more symptom-modifying } \\
\text { medication }\end{array}$ & $92(79.3)$ & $131(85.1)$ & $89(89.0)$ & $100(92.6)$ & 0.0272 \\
\hline
\end{tabular}

${ }^{\mathrm{a}} \mathrm{N}=478$ total external sample. Data are $n$ (\%). BMI, Body mass index; FIQ, Fibromyalgia Impact Questionnaire; FM, Fibromyalgia; SF-36, Medical Outcomes Study 36-item Short Form survey; SNRI, Serotonin norepinephrine reuptake inhibitor; SS, Symptom severity (SS); SSRI, Selective serotonin reuptake inhibitor; TCA, Tricyclic antidepressant; WPI, Widespread pain index. ${ }^{b}$ Low symptom intensity. ' Moderate symptoms, low negative mood. ${ }^{d}$ Moderate symptoms, higher negative mood and dyscognition. ${ }^{\mathrm{e}}$ High symptom intensity. ${ }^{\mathrm{f}} \mathrm{P}$-values are from the omnibus analysis of variance test across the four clusters. . ${ }^{\mathrm{g}}$ Lower scores are worse for these scales. ${ }^{\mathrm{h}}$ Includes other antidepressants, such as lithium, monoamine oxidase inhibitors, antipsychotics, bupropion, buspirone, lamotrigine and tetracyclic antidepressants. 
Medication use across clusters is described in Table 6. In regard to serotonin norepinephrine reuptake inhibitors (SNRIs) and $\alpha_{2} \delta$ ligands, classes that include the US Food and Drug Administration-approved medications for FM, cluster 4 had the highest percentage of patients taking both SNRIs (44\%) and $\alpha_{2} \delta$ ligands (22\%), in comparison to cluster 1 , which had $30 \%$ of patients receiving SNRIs and $20 \%$ taking $\alpha_{2} \delta$ ligands. Opioid use was significantly different across clusters, with $53 \%$ of patients in cluster 4 using opioids in comparison with $18 \%$ in cluster 1 . Similarly, use of benzodiazepines was different across clusters, with $49 \%$ of patients in cluster 3 and $44 \%$ in cluster 4 taking benzodiazepines, in comparison to $19 \%$ of patients in cluster 1 and $21 \%$ in cluster 2 .

\section{Discussion}

In the exploratory cluster analysis, we identified four unique clusters using self-report questionnaires representing eight core OMERACT symptom domains (fatigue, sleep disturbance, pain, function, stiffness, dyscognition, depression and anxiety). The four subgroups that were derived from this cluster analysis included (1) a generally low symptom intensity group (cluster 1); (2) a moderate symptom, low anxiety and low depression group (cluster 2); (3) a moderate symptom, higher anxiety and higher depression group (cluster 3); and (4) a generally high symptom intensity group (cluster 4 ). Despite the fact that the validation sample consisted of younger patients with higher FM symptom severity, the cluster solution in this sample was similar to the exploratory cluster analysis.

Our results share common themes with previous cluster analysis studies in patients with FM. For example, in the cluster analyses by Wilson et al. [10], Loevinger et al. [7] and de Souza et al. [5], there appear to be at least one subgroup of patients who have high physical and psychological symptoms (similar to our cluster 4) and one subgroup with less psychological distress and low levels of physical symptoms (similar to our cluster 1). Notably, our results are quite similar to those of Wilson et al. in that we also identified four clusters: one cluster that was low on all symptom domains, two clusters that were moderate on physical domains but distinguished by differences on psychological factors, and one cluster that was high on all symptom domains [10]. Although clusters 2 and 3 had overall moderate levels of symptoms, the severity of depression and anxiety was the distinguishing factor, which is consistent with the findings reported by Loevinger et al. [7] and de Souza et al. [5] in that psychological distress may be one factor that differentiates clusters.

Our preliminary cluster solution is further supported by FIQ-R and SF-36 scores, which serve as external benchmarks. Cluster 1 in both samples had the lowest mean FIQ-R total score, which also fell within the mild symptom severity range described for the FIQ-R (score 0 to $<39$ ) [55]. Compared to this finding, cluster 4 fell in the severe symptom severity range for the FIQ-R ( $\geq 59$ to 100). Similarly, cluster 1 in both samples had the best physical and mental composite scores on the SF-36, and score ranges appeared relatively close to healthy population norms $(50 \pm 10)$ [21]. Cluster 4 in both samples had the worst physical and mental composite scores, indicating the poorest levels of physical and mental health. Using disability status as yet another external benchmark of symptom burden and/or impact, we found that the percentage of patients on work disability significantly increased $(P<0.0001)$ as symptom severity increased across clusters.

We were not able to compare medication use across clusters in our exploratory sample (as medication use at the time of the survey was not available). When we compared medication use in the validation sample, however, cluster 1 had the lowest percentage using any medications for FM and cluster 4 had the highest percentage. Although this finding was somewhat surprising, it demonstrates that patients in cluster 4, despite being on multiple medications (patients in cluster 4 were taking an average of 3.4 medications each), had the highest level of symptom burden. This indicates that symptom severity in cluster 4 is not driven by the lack treatment, but rather it may be indicative of the symptom severity spectrum of FM.

In contrast to clusters 1 and 4, clusters 2 and 3 (both exploratory and validation samples) had similar levels of total FIQ-R symptom severity. The cluster differentiation between clusters 2 and 3 was driven primarily by anxiety and depression, with cluster 3 having significantly higher levels. Comparison of medication use in the validation sample indicated that there were no significant differences in medication use between clusters 2 and 3, except with regard to use of benzodiazepines, which was significantly higher in cluster $3(P<0.0001)$. This indicates that the differences in depression and anxiety that distinguished clusters 2 and 3 were not due to one cluster being differentially treated. In fact, patients in cluster 3 were taking more medications for anxiety (benzodiazepines) compared to cluster 2 and had more severe anxiety.

In support of these results, the SF-36 Mental scores indicated better mental health in cluster 2 compared to cluster 3 in both samples. However, the SF-36 Physical scores were higher in cluster 2 compared to cluster 3 in both samples. A reason for this might be that the increased BMI in cluster 2 could be associated with poorer physical function. Although BMI was significantly higher in cluster 2 in the exploratory sample, the difference in the validation sample did not reach statistical significance.

We recognize several limitations to this study. First, despite identification of four clusters, the sample is crosssectional and the longitudinal stability of these clusters is unknown. One published study on the longitudinal 
behavior of FM symptoms over the course of 5.5 years indicated that pain, fatigue and sleep demonstrated small longitudinal fluctuations over time at the group level, but showed significant within-patient variability [56]. A second limitation is that objective biomarkers recommended by OMERACT, such as tenderness, CSF biomarkers and functional imaging, were not available and therefore could not be included in the clustering. A third limitation is that, based on our inclusion criteria, we cannot comment on the generalizability of our results to community samples or men with FM, given our sample of female patients with FM identified through a clinical registry. Fourth, variables such as those included by Docampo et al. (sociodemographic characteristics, medications, comorbidities and personal or family history) that we were unable to include [11], and our questionnaire selection could influence clustering; however, the rationale for our use of these variables was based on the OMERACT recommendations, and care was taken to avoid any overlap between items representing symptom domains. Despite this, both our results and those of Docampo et al. suggest that varying symptom combinations are present in a heterogeneous sample of patients with FM.

\section{Conclusions}

Our study corroborates previous cluster reports, but also distinguishes itself by being the first study of its kind to incorporate a comprehensive spectrum of symptoms, as recommended by the OMERACT FM working group. We acknowledge that this cluster solution needs longitudinal study and that the feasibility and outcomes of this approach must be assessed before any conclusions regarding its clinical implications can be made; however, the present study is a first step toward this goal.

\section{Abbreviations}

BMI: Body mass index; BPI: Brief Pain Inventory; FIQ-R: Revised Fibromyalgia Impact Questionnaire; FM: Fibromyalgia; GAD-7: Generalized Anxiety Disorder 7-item scale; MASQ: Multiple Ability Self-report Questionnaire;

MFI: Multidimensional Fatigue Inventory; MOS-Sleep: Medical Outcomes Study Sleep Scale; OMERACT: Outcome Measures in Rheumatology; PHQ-9: Patient Health Questionnaire; POMS: Profile of Mood States; SF-36: 36-item Short Form survey; SNRI: Serotonin norepinephrine reuptake inhibitor; SS: Symptom severity; SSRI: Selective serotonin reuptake inhibitor; WPI: Widespread Pain Index.

\section{Competing interests}

The authors declare that they have no competing interests.

\section{Authors' contributions}

AV and TLH conceived of and designed the study, collected and analyzed data and wrote the manuscript. MOW collected and analyzed data and wrote the manuscript. DJC, DLB, RPB and DAW conceived of and designed the study and critically revised the manuscript. All authors read and approved the final manuscript.

\section{Acknowledgements}

This study was supported in part by the Center for Translational Science Activities (CTSA) at the Mayo Clinic. This center is funded in part by a grant from the National Center for Research Resources (NCRR), a component of the National Institutes of Health (NIH) (RR024150). The manuscript contents are solely the responsibility of the authors and do not necessarily represent the official views of CTSA, NCRR or NIH. The funders had no role in the study design, data collection and analysis, decision to publish or manuscript preparation. The study data were collected and managed using REDCap electronic data capture tools hosted at the Mayo Clinic. REDCap (Research Electronic Data Capture) is a secure, web-based application designed to support data capture for research studies by providing (1) an intuitive interface for validated data entry, (2) audit trails for tracking data manipulation and export procedures, (3) automated export procedures for seamless data downloads to common statistical packages and (4) procedures for importing data from external sources.

\section{Author details}

'Division of General Internal Medicine, Mayo Clinic, 200 First Street SW, Rochester, MN 55902, USA. ${ }^{2}$ Division of Biomedical Statistics and Informatics, Mayo Clinic, 200 First Street SW, Rochester, MN 55902, USA. ${ }^{3}$ Chronic Pain and Fatigue Research Center, University of Michigan Health System, 24 Frank Lloyd Wright Drive, Ann Arbor, MI 48105, USA. ${ }^{4}$ Division of Medical Oncology, Mayo Clinic, 200 First Street SW, Rochester, MN 55902, USA. ${ }^{5}$ Division of Pulmonary and Critical Care Medicine, Mayo Clinic, 200 First Street SW, Rochester, MN 55902, USA.

Received: 20 February 2014 Accepted: 23 September 2014

Published online: 16 October 2014

\section{References}

1. Fibromyalgia: poorly understood; treatments are disappointing. Prescrire Int 2009, 18:169-173.

2. Clauw DJ, Arnold LM, McCarberg BH, FibroCollaborative: The science of fibromyalgia. Mayo Clin Proc 2011, 86:907-911.

3. Wolfe F, Clauw DJ, Fitzcharles MA, Goldenberg DL, Katz RS, Mease P, Russell AS, Russell IJ, Winfield JB, Yunus MB: The American College of Rheumatology preliminary diagnostic criteria for fibromyalgia and measurement of symptom severity. Arthritis Care Res 2010, 62:600-610.

4. Bennett RM, Russell J, Cappelleri JC, Bushmakin AG, Zlateva G, Sadosky A Identification of symptom and functional domains that fibromyalgia patients would like to see improved: a cluster analysis. BMC Musculoskelet Disord 2010, 11:134.

5. de Souza JB, Goffaux P, Julien N, Potvin S, Charest J, Marchand S: Fibromyalgia subgroups: profiling distinct subgroups using the Fibromyalgia Impact Questionnaire: a preliminary study. Rheumatol Int 2009, 29:509-515.

6. Giesecke T, Williams DA, Harris RE, Cupps TR, Tian X, Tian TX, Gracely RH, Clauw DJ: Subgrouping of fibromyalgia patients on the basis of pressure-pain thresholds and psychological factors. Arthritis Rheum 2003, 48:2916-2922.

7. Loevinger BL, Shirtcliff EA, Muller D, Alonso C, Coe CL: Delineating psychological and biomedical profiles in a heterogeneous fibromyalgia population using cluster analysis. Clin Rheumatol 2012, 31:677-685.

8. Rutledge DN, Mouttapa M, Wood PB: Symptom clusters in fibromyalgia: potential utility in patient assessment and treatment evaluation. Nurs Res 2009, 58:359-367.

9. Verra ML, Angst F, Brioschi R, Lehmann S, Keefe FJ, Staal JB, de Bie RA, Aeschlimann A: Does classification of persons with fibromyalgia into Multidimensional Pain Inventory subgroups detect differences in outcome after a standard chronic pain management program? Pain Res Manag 2009, 14:445-453.

10. Wilson HD, Robinson JP, Turk DC: Toward the identification of symptom patterns in people with fibromyalgia. Arthritis Rheum 2009, 61:527-534.

11. Docampo E, Collado A, Escaramís G, Carbonell J, Rivera J, Vidal J, Alegre J, Rabionet $R$, Estivill $X$ : Cluster analysis of clinical data identifies fibromyalgia subgroups. PLoS One 2013, 8:e74873.

12. Turk DC, Okifuji A, Sinclair JD, Starz TW: Differential responses by psychosocial subgroups of fibromyalgia syndrome patients to an interdisciplinary treatment. Arthritis Care Res 1998, 11:397-404.

13. Mease PJ, Arnold LM, Crofford LJ, Williams DA, Russell IJ, Humphrey L, Abetz L, Martin SA: Identifying the clinical domains of fibromyalgia: contributions from clinician and patient Delphi exercises. Arthritis Rheum 2008, 59:952-960.

14. Whipple MO, McAllister SJ, Oh TH, Luedtke CA, Toussaint LL, Vincent A: Construction of a US fibromyalgia registry using the Fibromyalgia Research Survey criteria. Clin Trans Sci 2013, 6:398-399.

15. Cleeland CS, Ryan KM: Pain assessment: global use of the Brief Pain Inventory. Ann Acad Med Singapore 1994, 23:129-138. 
16. McNair DM, Lorr M, Droppleman LF: EdITS Manual for the Profile of Mood States. San Diego, CA: Educational and Industrial Testing Service; 1992.

17. Cappelleri JC, Bushmakin AG, McDermott AM, Dukes E, Sadosky A, Petrie CD, Martin S: Measurement properties of the medical outcomes study sleep scale in patients with fibromyalgia. Sleep Med 2009, 10:766-770.

18. Smets EM, Garssen B, Bonke B, De Haes JC: The Multidimensional Fatigue Inventory (MFI) psychometric qualities of an instrument to assess fatigue. J Psychosom Res 1995, 39:315-325.

19. Seidenberg M, Haltiner A, Taylor MA, Hermann BB, Wyler A: Development and validation of a Multiple Ability Self-Report Questionnaire. Clin Exp Neuropsychol 1994, 16:93-104.

20. Bennett RM, Friend R, Jones KD, Ward R, Han BK, Ross RL: The Revised Fibromyalgia Impact Questionnaire (FIQR): validation and psychometric properties. Arthritis Res Ther 2009, 11:R120.

21. Ware JE Jr: SF-36 health survey update. Spine 2000, 25:3130-3139.

22. Williams DA, Arnold LM: Measures of fibromyalgia: Fibromyalgia Impact Questionnaire (FIQ), Brief Pain Inventory (BPI), Multidimensional Fatigue Inventory (MFI-20), Medical Outcomes Study (MOS) Sleep Scale, and Multiple Ability Self-Report Questionnaire (MASQ). Arthritis Care Res 2011, 63:S86-S97.

23. Wolfe F, Clauw DJ, Fitzcharles MA, Goldenberg DL, Häuser W, Katz RS, Mease P, Russell AS, Russell IJ, Winfield JB: Fibromyalgia criteria and severity scales for clinical and epidemiological studies: a modification of the ACR Preliminary Diagnostic Criteria for Fibromyalgia. J Rheumatol 2011, 38:1113-1122.

24. Aparicio VA, Ortega FB, Carbonell-Baeza A, Femia P, Tercedor P, Ruiz JR, Delgado-Fernández M: Are there gender differences in quality of life and symptomatology between fibromyalgia patients? Am J Mens Health 2012 , 6:314-319.

25. Buskila D, Neumann L, Alhoashle A, Abu-Shakra M: Fibromyalgia syndrome in men. Semin Arthritis Rheum 2000, 30:47-51.

26. Castro-Sánchez AM, Matarán-Peñarrocha GA, López-Rodríguez MM, Lara-Palomo IC, Arendt-Nielsen L, Fernández-de-las-Peñas C: Gender differences in pain severity, disability, depression, and widespread pressure pain sensitivity in patients with fibromyalgia syndrome without comorbid conditions. Pain Med 2012, 13:1639-1647.

27. Hooten WM, Townsend CO, Decker PA: Gender differences among patients with fibromyalgia undergoing multidisciplinary pain rehabilitation. Pain Med 2007, 8:624-632.

28. Mease P, Arnold LM, Choy EH, Clauw DJ, Crofford LJ, Glass JM, Martin SA Morea J, Simon L, Strand CV, Williams DA: Fibromyalgia syndrome module at OMERACT 9: domain construct. J Rheumatol 2009, 36:2318-2329.

29. Cleeland CS: The Brief Pain Inventory: User Guide. Houston, TX: Charles S Cleeland; 2009. [http://www.mdanderson.org/education-and-research/departmentsprograms-and-labs/departments-and-divisions/symptom-research/symptomassessment-tools/BPI UserGuide.pdf] (accessed 1 November 2014).

30. Arnold LM, Rosen A, Pritchett YL, D'Souza DN, Goldstein DJ, lyengar S, Wernicke JF: A randomized, double-blind, placebo-controlled trial of duloxetine in the treatment of women with fibromyalgia with or without major depressive disorder. Pain 2005, 119:5-15.

31. Chappell AS, Bradley LA, Wiltse C, Detke MJ, D'Souza DN, Spaeth M: A six-month double-blind, placebo-controlled, randomized clinical trial of duloxetine for the treatment of fibromyalgia. Int J Gen Med 2008, 1:91-102.

32. Mease PJ, Spaeth M, Clauw DJ, Arnold LM, Bradley LA, Russell IJ, Kajdasz DK, Walker DJ, Chappell AS: Estimation of minimum clinically important difference for pain in fibromyalgia. Arthritis Care Res 2011, 63:821-826.

33. Russell IJ, Mease PJ, Smith TR, Kajdasz DK, Wohlreich MM, Detke MJ, Walker DJ, Chappell AS, Arnold LM: Efficacy and safety of duloxetine for treatment of fibromyalgia in patients with or without major depressive disorder: Results from a 6-month, randomized, double-blind, placebo-controlled, fixed-dose trial. Pain 2008, 136:432-444.

34. Bourgeois A, LeUnes A, Meyers M: Full-scale and short-form of the Profile of Mood States: a factor analytic comparison. J Sport Behav 2010, 33:355-376.

35. Malin K, Littlejohn GO: Psychological control is a key modulator of fibromyalgia symptoms and comorbidities. J Pain Res 2012, 5:463-471.

36. Katz RS, Heard AR, Mills M, Leavitt F: The prevalence and clinical impact of reported cognitive difficulties (fibrofog) in patients with rheumatic disease with and without fibromyalgia. J Clin Rheumatol 2004, 10:53-58.

37. Arnold LM, Russell IJ, Diri EW, Duan WR, Young JP Jr, Sharma U, Martin SA, Barrett JA, Haig G: A 14-week, randomized, double-blinded, placebocontrolled monotherapy trial of pregabalin in patients with fibromyalgia. J Pain 2008, 9:792-805.
38. Crofford LJ, Rowbotham MC, Mease PJ, Russell IJ, Dworkin RH, Corbin AE, Young JP Jr, LaMoreaux LK, Martin SA, Sharma U, Pregabalin 1008-105 Study Group: Pregabalin for the treatment of fibromyalgia syndrome: results of a randomized, double-blind, placebo-controlled trial. Arthritis Rheum 2005, 52:1264-1273.

39. Martin S, Chandran A, Zografos L, Zlateva G: Evaluation of the impact of fibromyalgia on patients' sleep and the content validity of two sleep scales. Health Qual Life Outcomes 2009, 7:64

40. Lin JMS, Brimmer DJ, Maloney EM, Nyarko E, BeLue R, Reeves WC: Further validation of the Multidimensional Fatigue Inventory in a US adult population sample. Popul Health Metr 2009, 7:18.

41. Clauw DJ, Mease P, Palmer RH, Gendreau RM, Wang Y: Milnacipran for the treatment of fibromyalgia in adults: a 15-week, multicenter, randomized, double-blind, placebo-controlled, multiple-dose clinical trial. Clin Ther 2008, 30:1988-2004.

42. Arnold LM, Wang F, Ahl J, Gaynor PJ, Wohlreich MM: Improvement in multiple dimensions of fatigue in patients with fibromyalgia treated with duloxetine: Secondary analysis of a randomized, placebo-controlled trial. Arthritis Res Ther 2011, 13:R86.

43. Owen RT: Milnacipran hydrochloride: its efficacy, safety and tolerability profile in fibromyalgia syndrome. Drugs Today (Barc) 2008, 44:653-660.

44. Branco JC, Bannwarth B, Failde I, Abello Carbonell J, Blotman F, Spaeth M, Saraiva F, Nacci F, Thomas E, Caubère JP, Le Lay K, Taieb C, Matucci-Cerinic M: Prevalence of fibromyalgia: a survey in five European countries. Semin Arthritis Rheum 2010, 39:448-453.

45. Mease PJ, Clauw DJ, Gendreau RM, Rao SG, Kranzler J, Chen W, Palmer RH: The efficacy and safety of milnacipran for treatment of fibromyalgia: a randomized, double-blind, placebo-controlled trial. J Rheumatol 2009, 36:398-409. A published erratum appears in J Rheumatol 2009, 36:661.

46. Arnold LM, Hess EV, Hudson Jl, Welge JA, Berno SE, Keck PE Jr: A randomized, placebo-controlled, double-blind, flexible-dose study of fluoxetine in the treatment of women with fibromyalgia. Am J Med 2002, 112:191-197.

47. Arnold LM, Gendreau RM, Palmer RH, Gendreau JF, Wang Y: Efficacy and safety of milnacipran $100 \mathrm{mg} /$ day in patients with fibromyalgia: results of a randomized, double-blind, placebo-controlled trial. Arthritis Rheum 2010, 62:2745-2756.

48. Da Costa D, Dobkin PL, Fitzcharles MA, Fortin PR, Beaulieu A, Zummer M, Senécal JL, Goulet JR, Rich E, Choquette D, Clarke AE: Determinants of health status in fibromyalgia: a comparative study with systemic lupus erythematosus. J Rheumatol 2000, 27:365-372

49. Neumann L, Berzak A, Buskila D: Measuring health status in Israeli patients with fibromyalgia syndrome and widespread pain and healthy individuals: utility of the Short Form 36-item health survey (SF-36). Semin Arthritis Rheum 2000, 29:400-408.

50. Tüzün EH, Albayrak G, Eker L, Sözay S, Daşkapan A: A comparison study of quality of life in women with fibromyalgia and myofascial pain syndrome. Disabil Rehabil 2004, 26:198-202.

51. Löwe B, Decker O, Müller S, Brähler E, Schellberg D, Herzog W, Herzberg PY: Validation and standardization of the Generalized Anxiety Disorder Screener (GAD-7) in the general population. Med Care 2008, 46:266-274

52. Spitzer RL, Kroenke K, Williams JBW, Löwe B: A brief measure for assessing generalized anxiety disorder: the GAD-7. Arch Intern Med 2006, 166:1092-1097.

53. Smarr KL, Keefer AL: Measures of depression and depressive symptoms: Beck Depression Inventory-II (BDI-II), Center for Epidemiologic Studies Depression Scale (CES-D), Geriatric Depression Scale (GDS), Hospital Anxiety and Depression Scale (HADS), and Patient Health Questionnaire-9 (PHQ-9). Arthritis Care Res 2011, 63:S454-S466.

54. R Development Core Team: R: A Language and Environment for Statistical Computing. Vienna, Austria: R Foundation for Statistical Computing; 2012

55. Schaefer C, Chandran A, Hufstader M, Baik R, McNett M, Goldenberg D, Gerwin R, Zlateva G: The comparative burden of mild, moderate and severe fibromyalgia: results from a cross-sectional survey in the United States. Health Qual Life Outcomes 2011, 9:71

56. Walitt B, Fitzcharles MA, Hassett AL, Katz RS, Häuser W, Wolfe F: The longitudinal outcome of fibromyalgia: a study of 1555 patients. J Rheumatol 2011, 38:2238-2246.

doi:10.1186/s13075-014-0463-7

Cite this article as: Vincent et al:: OMERACT-based fibromyalgia symptom subgroups: an exploratory cluster analysis. Arthritis Research \& Therapy 2014 16:463. 